\title{
EVALUATION OF ANTIBACTERIAL ACTIVITY IN EXPERIMENTAL MENINGO-ENCEPHALITIS IN MICE*
}

\author{
F. Campanile, M. C. Fioretti, E. Bonmassar ${ }^{\dagger}$ and P. Puccetti \\ Institute of Pharmacology, University of Perugia, \\ Via del Giochetto, 06100 Perugia, Italy \\ 'Department of Experimental Medicine, 2 Rome University, \\ Via Orazio Raimondo, Tor Vergata, 00173 Roma, Italy
}

(Received for publication March 27, 1985)

\begin{abstract}
Mice infected intracerebrally (ic) or intraperitoneally (ip) with Pseudomonas aeruginosa were treated subcutaneously with graded doses of five antibiotics all of which displayed antipseudomonal activity in vitro. The analysis of $\mathrm{MIC}, \mathrm{ED}_{50}$ in the ip infection model ( $\mathrm{ED}_{50}$-ip) and that in the ic system $\left(E D_{50}-i c\right)$ for each agent showed: a) gentamicin, the most active agent in vitro, was also active in vivo, with an $\mathrm{ED}_{50}$-ic nearly six-fold higher than $\mathrm{ED}_{50}$-ip; b) ceftazidime, considerably active both in vitro and in vivo, showed comparable values of $\mathrm{ED}_{50}$ in the two systems; c) ceftriaxone, also active in vitro, showed limited activity in vivo, its two $\mathrm{ED}_{50}$ values being similar; d) aztreonam, moderately active both in vitro and in vivo, showed comparable activity in the two infection models; e) carbenicillin, the least active agent in vitro, gave poor results in vivo, with an $\mathrm{ED}_{50}$-ic 2.6-fold higher than $\mathrm{ED}_{50}$-ip.
\end{abstract}

Despite the introduction of new antimicrobial agents and a more thorough understanding of the pathology of infections of the central nervous system, the mortality and morbidity of bacterial meningitis and brain abscess remain high. In this respect, Pseudomonas aeruginosa is undoubtedly a Gramnegative pathogen of major importance as it frequently causes fatal sepsis and/or localized lesions in areas or individuals particulary devoid of defense ${ }^{1)}$. Because of the varied results obtained with aminoglycoside treatment in Gram-negative bacillary meningitis ${ }^{2,3)}$, there is a need for continued development and screening of new antimicrobial agents for therapy of Pseudomonas infections.

In the present study we analyzed the antipseudomonal activity of different agents in mice and, for each antibiotic, the therapeutic efficacy $\left(\mathrm{ED}_{50}\right)$ in experimental meningo-encephalitis was compared to that in peritonitis. This type of analysis was found to be suitable for identifying different patterns of activity among the tested drugs.

\section{Materials and Methods}

Mice

$\overline{\mathrm{Balb}} / \mathrm{c}$ female mice, $6 \sim 10$ weeks of age, were obtained from the Mammalian Genetics and Animal Production Section, Division of Cancer Treatment, National Cancer Institute (NCI), National Institutes of Health (NIH), Bethesda, MD, USA.

\section{Organism}

A single strain of $P$. aeruginosa, immunotype $11^{4,5)}$, isolated from a clinical specimen was supplied by Dr. CASSONE, Istituto Superiore di Sanità, Roma. The organism was routinely cultured in nutrient broth (Difco) and incubated at $37^{\circ} \mathrm{C}$ for 18 to 24 hours with constant aeration.

Preparation of Inoculum

The cultures were centrifuged at 15,000 rpm for 15 minutes in a Sorvall RC 2 B centrifuge, the supernatant was discarded, and the soft pellet of cells was resuspended in sterile saline. Viable cell

* This work was supported by CNR-Rome grant No. 83.00630.52. 
count was performed by the colony-forming unit method using Tryptose agar. A standard curve, based on turbidity as read on a photoelectric colorimeter, was also prepared to correlate turbidity with viable colony counts, and was used subsequently to determine challenge concentrations.

Induction of Meningo-encephalitis

The intracerebral (ic) inoculum procedure was that described by CAMPANILE et al..$^{45}$. $P$. aeruginosa cells were suspended in $0.03 \mathrm{ml}$ saline aliquot and injected under sterile conditions through the central-middle area of the frontal bone to $2-\mathrm{mm}$ depth by means of $0.1 \mathrm{ml}$ glass microsyringe and 27-gauge disposable needle. All animals developed meningo-encephalitis as proved by clinical signs and histopathological examination. Control mice receiving saline with no bacteria fully recovered from trauma within $30 \sim 60$ minutes. Surgical mortality was less than $3 \%$ and always occurred within $1 \sim 2$ minutes from injection.

\section{Induction of Peritonitis}

The animals were injected intraperitoneally (ip) with $0.2 \mathrm{ml}$ of the appropriate dilution of $P$. aeruginosa in saline. All animals developed peritonitis as proved by clinical signs and histopathological examination.

\section{Determination of $\mathrm{LD}_{50}$}

Fifty percent lethal dose $\left(\mathrm{LD}_{50}\right)$ values were determined from the results of ip or ic administration of graded doses of live organisms and calculated by the method of LitCHFIELD and WILCOXON ${ }^{6}$.

\section{Antibiotics}

Gentamicin (Gentalyn, Essex) and carbenicillin (Geopen, Pfizer) were purchased. Ceftazidime (RG 159, Glaxo), aztreonam (SQ 26776, Squibb) and ceftriaxone (Rocefin, Roche) were kindly supplied by their respective manufacturers. Ceftazidime was prepared as the pentahydrate and converted to the sodium salt by dissolution in sodium carbonate. Aztreonam was supplied in vials as a sterile powder blend of aztreonam and L-arginine for reconstitution with $10 \mathrm{ml}$ sterile water for injection (weight ratio of aztreonam to L-arginine 1.0/0.78). Ceftriaxone was provided as the disodium salt to be reconstituted with sterile water for injection.

\section{Susceptibility Testing}

Minimum inhibitory concentrations (MICs) were determined by a broth microdilution method using nutrient broth (Difco). $10^{4}$ organisms $/ 0.1 \mathrm{ml}$ (four replicates) were placed in the wells of microdilution plates and brought to a final volume of $0.2 \mathrm{ml}$ by addition of serial two-fold dilutions of antibiotics in nutrient broth (range of final concentrations: $0.03 \sim 125 \mu \mathrm{g} / \mathrm{ml}$ ). The plates were incubated for 24 hours at $37^{\circ} \mathrm{C}$. The MIC was taken as the highest dilution of antimicrobial agent in which no growth appeared.

\section{Mouse Protection Test}

The infected animals were treated subcutaneously (sc) at 1 and 5 hours from bacterial challenge with serial two-fold dilutions of each antibiotic. $6 \sim 8$ mice were used at each dose level and deaths were recorded for 5 days. Mean effective doses of antibiotics $\left(E D_{50}\right)$ in the protection experiments were calculated according to the method of LITCHFIELD and WILCOXON ${ }^{6)}$, using experimental groups of $6 \sim 8$ animals.

\section{Results}

\section{Meningo-encephalitis Model}

The object of the present study was to analyze the penetration of different antibiotics into the cerebrospinal fluid (CSF) of mice infected ic with $P$. aeruginosa by comparing the mean effective dose of each agent in the ic infection model with that in the ip system. The working hypothesis was that, provided the two models were similar with respect to mortality criteria, the $\mathrm{ED}_{50}$-ic would relate to 
Table 1. Mortality data of mice challenged with Pseudomonas aeruginosa.

\begin{tabular}{ccccc}
\hline Mice injected & Route & $\begin{array}{c}\text { Inoculum size } \\
\left(\times 10^{\mathrm{c}} \mathrm{cfu}\right)\end{array}$ & $\begin{array}{c}\text { Mortality } \\
(\%)\end{array}$ & $\begin{array}{c}\text { MST }^{\mathrm{c}} \\
(\text { (range })\end{array}$ \\
\hline $44^{\mathrm{a}}$ & ic & 1.5 & 94.4 & $1 \sim 3$ \\
$68^{\mathrm{b}}$ & ip & 100 & 96.6 & $1 \sim 3$ \\
\hline
\end{tabular}

a Data from 7 experiments. b Data from 10 experiments. c Median survival time (days).

Table 2. Mouse protection test with different antibiotics in Pseudomonas aeruginosa infections.

\begin{tabular}{lcccc}
\hline Antibiotic & $\mathrm{MIC}^{\mathrm{a}}$ & $\mathrm{ED}_{50} \mathrm{ic}^{\mathrm{b}}$ & $\mathrm{ED}_{50}$-ip $^{\mathrm{b}}$ & $\mathrm{ED}_{50} \mathrm{ic}^{\mathrm{ic}} / \mathrm{ED}_{50}-\mathrm{ip}$ \\
\hline Gentamicin & $<0.03$ & $90(82 \sim 99)^{\mathrm{c}}$ & $16(11 \sim 24)$ & $5.6^{\mathrm{d}}$ \\
Ceftazidime & 0.97 & $32(22 \sim 49)$ & $37(26 \sim 52)$ & 0.8 \\
Ceftriaxone & 0.97 & $500(370 \sim 675)$ & $450(281 \sim 720)$ & 1.1 \\
Aztreonam & 3.9 & $152(101 \sim 227)$ & $106(68 \sim 165)$ & 1.4 \\
Carbenicillin & 31.2 & $1,285(955 \sim 1,728)$ & $480(266 \sim 864)$ & 2.6 \\
\hline
\end{tabular}

a $\mu \mathrm{g} / \mathrm{ml}, \quad$ b $\mathrm{mg} / \mathrm{kg}, \quad$ c $95 \%$ confidence intervals, d numbers are $\mathrm{ED}_{50}$-ic to $\mathrm{ED}_{50}$-ip ratios.

$\mathrm{ED}_{50}$-ip in a fashion largely conditioned by the agent pharmacokinetics, regardless of its absolute anti-Pseudomonas activity. When Balb/c female mice were injected ic with graded numbers of $P$. aeruginosa cells, the mean lethal dose $\left(\mathrm{LD}_{50}\right)$ was found to be $3 \times 10^{6}$ cells/mouse. At least $50 \mathrm{LD}_{50}$ were needed to obtain a percent mortality of nearly $95 \%$ (data pooled from 7 experiments, Table 1). In individual experiments, the median survival time (MST) of mice receiving $50 \mathrm{LD}_{50}$ was $1.5 \sim 2$ days. When $P$. aeruginosa was injected into the peritoneal cavity, the $\mathrm{LD}_{50}$ was found to be $10^{\circ}$ cells $/ \mathrm{mouse}$. Ten $\mathrm{LD}_{50}$ would result in a percent mortality of approximately $97 \%$ with an MST in most experiments of 2 days (Table 1). In both models, all deaths resulting from infection occurred within 5 days from microbial challenge.

\section{In Vitro and In Vivo Activity of Different Antibiotics}

Gentamicin, ceftazidime, ceftriaxone, aztreonam and carbenicillin are antibiotics with antiPseudomonas activity and have long been used in, or are currently proposed for, the treatment of Gram-negative bacterial meningitis. Balb/c mice were challenged ic ( $50 \mathrm{LD}_{50}$ units) or ip (10 LD $\mathrm{L}_{50}$ units) with $P$. aeruginosa. The animals were then treated twice (at 1 and 5 hours, subcutaneously) with graded doses of each antibiotic. Deaths were recorded for 5 days and fifty percent effective dose values $\left(E D_{50}\right.$-ic and $\mathrm{LD}_{50}$-ip) of each agent in the 2 systems were calculated. Minimum inhibitory concentrations in vitro were obtained as previously described. Table 2 summarizes the results.

\section{Discussion}

The present studies describe a model of meningo-encephalitis in mice which is a reliable means for assaying antibacterial activity in infections of the central nervous system. One advantage of this model is that, by comparatively analyzing the therapeutic activity of an agent in the ic and ip systems, it is possible to high-light the impact of pharmacokinetics in the drug activity in the ic infection. As a reference infection we selected, in this study, acute peritonitis, sustained by a high bacterial inoculum, so as to provide a disease with a course as rapid as that of the ic infection. This resulted in comparable mortality rates in the two models. In general, however, the Pseudomonas strain used in this study displayed a relatively low virulence, possibly because of its being cultured extensively on laboratory medium ${ }^{7)}$. This may account for the lower $\mathrm{LD}_{50}$ values reported in the past by authors who employed recent clinical isolates of $P$. aeruginos $a^{8,9,10,11)}$. On the other hand, although in most studies lower 
inocula of $P$. aeruginosa were used, there is apparently no consensus as to the best bacterial inoculum for mouse protection test ${ }^{8}$. Moreover, while fluctations in the size of bacterial inoculum by a given route may result in variations of the $\mathrm{ED}_{50}$ of an antibiotic ${ }^{8,12)}$, the difference in the bacterial load in the ic and ip models is unlikely to have played a major role in our results.

Gentamicin was highly active in vitro against the strain of $P$. aeruginosa used in this study, which was reflected in vivo by an $\mathrm{ED}_{50}$-ip value of $16 \mathrm{mg} / \mathrm{kg}$, a finding in line with previous data in the literature $^{8)}$. A recent study on the penetration of antibiotics in CFS of rabbits with experimental meningitis proved the gentamicin concentration in CFS to be $21 \%$ of that found in serum following sc administration $^{13)}$. Our results indicate that doses of gentamicin nearly six-fold higher are needed to achieve protective effects in the ic model comparable to those in the ip system. Ceftazidime was also highly active both in vitro and in vivo, a finding in agreement with previous studies ${ }^{14)}$. Although little is known of the passage of ceftazidime into the CFS especially of animals with experimental meningitis, one might anticipate from our results that the percent penetration of the antibiotic should be sufficiently high. In fact, in our model the $\mathrm{ED}_{50}$-ic/ED $/ \mathrm{ED}_{50}$-ip ratio was 0.86. Ceftriaxone, although as effective in vitro as ceftazidime, gave poorer results in vivo. Nevertheless, the $\mathrm{ED}_{50}$-ic/ED $\mathrm{ED}_{50}$-ip ratio was 1.1, thus providing indirect evidence that ceftriaxone can penetrate cerebrospinal fluid effectively. In previous studies based on different meningo-encephalitis models, other authors reported essentially similar results ${ }^{15,16,17)}$. Aztreonam was moderately active both in vitro and in vivo. A recent study compared aztreonam with frequently used antibiotics in the therapy of three types of experimental bacterial meningitis in rabbits and in experimental Escherichia coli cerebritis in rats ${ }^{13)}$. Aztreonam was more active than the comparison agent in all models of experimental meningitis tested. The concentration of the antibiotic into purulent CFS was $23 \%$ of that in serum, among the highest for $\beta$-lactam antibiotics. Our findings indicate that the $\mathrm{ED}_{50}$-ic/ED $/ \mathrm{E}_{50}$-ip ratio is 1.4 for aztreonam.

Finally, carbenicillin, the least active agent in vitro on an absolute basis and progenitor of the $\beta$-lactam antibiotics with anti-pseudomonal activity, required doses in the ic model 2.6 times higher than those in the ip system to bring about comparable therapeutic results.

In conclusion, the studies summarized in the present paper my be useful for development of standardized models of meningo-encephalitis in mice for continued production and screening of antimicrobial agents effective in Gram-negative bacillary meningitis. Furthermore, our findings suggest that the newer $\beta$-lactam antibiotics with in vitro activity against members of the family Enterobacteriaceae may prove useful in infections of the central nervous system.

\section{References}

1) Cross, A.; J. R. Allen, J. Burke, G. Ducel, A. Harris, J. John, D. Johnson, M. Lew, B. MacMikan, P. Meers, et al.: Nosocomial infections due to Pseudomonas aeruginosa: a review of recent trends. Rev. Infection Dis. 5, Suppl. 5: S837 S845, 1983

2) Cherubin, C. E.; J. S. Marr, M. F. Sierra \& S. Beker: Listeria and Gram-negative bacillary meningitis in New York City, 1972 1979. Frequent causes of meningitis in adults. Am. J. Med. 77: 199 209, 1981

3) McCracken, G. H., Jr.; S. G. Mize \& N. Threlkeld: Intraventricular gentamicin therapy in Gramnegative bacillary meningitis of infancy. Lancet i: 787 791, 1980

4) Campanile, F.; P. Puccetti, G. Angeli, M. C. Fioretti \& E. Bonmassar: Experimental studies on mice challenged intracerebrally with Pseudomonas aeruginosa. Int. J. Tissue React. VI: 421 426, 1984

5) Campanile, F.; P. Puccetti, G. Angeli, M. C. Fioretti \& E. Bonmassar: Sulla messa a punto di un modello sperimentale di infezione meningo-encefalica. L'Igiene moderna 83: 154 162, 1985

6) Litchfield, J. T. \& F. Wilcoxon: A single method of evaluating dose effect experiments. J. Pharmacol. Exp. Ther. 4: 247 254, 1949

7) Cox, C. D.: Passage of Pseudomonas aeruginosa in compromised mice. Infect Immun. 26: 118 124, 1979

8) DAVIS, S. D.: Activity of gentamicin, tobramicin, polymyxin B and colistimethate in mouse protection test with Pseudomonas aeruginosa. Antimicrob. Agents Chemother. 8: 50 53, 1975

9) GaYdos, J. M.; L. Carrick, Jr. \& R. S. BerK: Experimental studies on mice challenged subcutaneously with Pseudomonas aeruginosa (38952). Proc. Soc. Exp. Biol. Med. 149: 908 914, 1975 
10) Pier, G. B.; H. F. Sidberry \& J. C. Sadoff: Protective immunity induced in mice by immunization with high-molecular-weight polysaccharide from Pseudomonas aeruginosa. Infect. Immun. 22: 919 925, 1978

11) Pierson, C. L.; A. G. Johnson \& I. Feller: Effect of cyclophosphamide on the immune response to Pseudomonas aeruginosa in mice. Infect. Immun. 14: 168 177, 1976

12) Acred, P.; P. A. Hunter, L. Mizen \& G. N. Rolinson: Carbenicillin: evaluation of antibacterial activity in vivo. Proc. 6th Int. Cong Chemother. 1: 305 308, 1970

13) Scheld, W. M.; J. P. Brodeur, J. C. Gratz, P. Foresman \& G. Rodeheaver: Evaluation of aztreonam in experimental bacterial meningitis and cerebritis. Antimicrob. Agents Chemother. 24: 682 688, 1983

14) ACRED, P.: Therapeutic and kinetic properties of ceftazidime in animals. Infection 11 (Suppl. 1): S44 $\mathrm{S} 48,1983$

15) Beskid, G.; J. G. Christenson, R. Cleeland, W. Delorenzo \& P. W. Trown: In vivo activity of ceftriaxone (Ro 13-9904), a new broad-spectrum semisynthetic cephalosporin. Antimicrob. Agents Chemother. 20: 159 167, 1981

16) Decazes, J. M.; J. D. ERnest \& M. A. SAnde: Correlation of in vitro time-kill curves and kinetics of bacterial killing in cerebrospinal fluid during ceftriaxone therapy of experimental Escherichia coli meningitis. Antimicrob. Agents Chemother. 24: 463 467, 1983

17) Marchon, B.; T. Van Tho \& M. Armengaud: Diffusion of ceftriaxone (Ro 13-9904/001) in the cerebrospinal fluid. Chemotherapy 27 (Suppl. 1): S37 S41, 1981 